\title{
Contact angle-based predictive model for slip at the solid-liquid interface of a transverse-shear mode acoustic wave device
}

\author{
Jonathan S. Ellis \\ Institute of Biomaterials and Biomedical Engineering, University of Toronto, 4 Taddle Creek Road, Toronto, \\ Ontario M5S 3G9, Canada \\ Glen McHale \\ School of Science, The Nottingham Trent University, Clifton Lane, Nottingham NG11 8NS, United Kingdom \\ Gordon L. Hayward \\ School of Engineering, University of Guelph, Guelph, Ontario N1G 2W1, Canada \\ Michael Thompson \\ Department of Chemistry, University of Toronto, 80 St. George Street, Toronto, Ontario M5S 3H6, Canada
}

(Received 23 June 2003; accepted 27 August 2003)

\begin{abstract}
We have revisited the Blake-Tolstoi theory [Coll. Surf. 47, 135 (1990)] for molecular and hydrodynamic slip and applied it to the fundamental description of acoustic wave devices coupled to a liquid of finite thickness. The aim is to provide a framework for a predictive model for slip, based on surface-liquid interactions and contact angle. This theory provides a description of slip that links hydrodynamic boundary slip to a schematic, molecular description involving the wettability of the liquid-solid interface. We redevelop the model, using current acoustic sensors notation, then evaluate its qualitative behavior as a predictive model for slip length in the context of acoustic wave devices. Finally, we discuss the limitations of the model and consider the advantages of a predictive model for boundary slip. (C) 2003 American Institute of Physics.
\end{abstract}

[DOI: $10.1063 / 1.1619195]$

\section{INTRODUCTION}

The thickness-shear mode acoustic wave device (TSM) has found rapidly increasing use as a sensor for biochemical species present in the aqueous phase. ${ }^{1}$ The principle of detection lies in the binding of a particular analyte to a chemically selective receptor moiety, which is attached to the surface of the device. This technique has its origins in the work of Sauerbrey, ${ }^{2}$ who related changes in the series resonant frequency to changes in surface-attached mass. In this treatment, any rigidly attached mass (in this case stemming from the gas phase) was assumed to possess the acoustic properties of the piezoelectric material employed for the fabrication of the sensor. In the liquid phase, the device is operated at high frequencies, normally in the megahertz range, over very small displacements $(3-10 \AA)$. As a result of the high speeds involved, complex hydrodynamic behavior may exist at the surface-liquid interface. The surface experiences shear rates on the order of $\dot{\gamma}=(d v / d x)_{z=0}=1000 \mathrm{~s}^{-1}$, which corresponds to the high shear regime and may result in strong slip. $^{3,4}$

There are varied interpretations of boundary slip, depending on the application. Slip can be seen as a discontinuity in the equations of motion at an interface, which are invoked through boundary conditions. This is known as hydrodynamic slip and has been applied in various forms across a wide range of areas, most notably in describing the flow of polymer melts. Most of the early advances in this field are attributed to de Gennes and co-workers. ${ }^{5-8}$ Another interpretation is molecular slip, which describes the motion of individual particles close to an interface. Israelachvili, ${ }^{9,10}$ describes stick-slip motion, which involves the dynamics of sliding friction between surfaces. Blake and co-workers ${ }^{11-13}$ have used molecular kinetics to characterize droplet spreading and hydrodynamic wetting.

A common hydrodynamic model of slip is the slip length boundary condition, ${ }^{3}$ which describes a vertical shift in the velocity profile so that the no-slip point occurs at some distance, a slip length $b$, within the solid surface, and there is a finite liquid velocity at the wall, as shown in Fig. 1. This description has been used extensively in describing the slip of polymer melts and in the explanation of observations of drainage flow in the surface force apparatus (SFA). For the slip length, shear rate is a strong determinant as to whether slip will occur. ${ }^{14,15}$ At small shear rates, molecular ordering of a liquid near a wall is strong enough to retain the liquid molecules at the wall, which is the no-slip condition. However, as the shear rate of the liquid is increased, the shear stresses in the liquid become strong enough to overcome the ordering induced by the wall, and motion of the molecules adjacent to the wall may increase. At lower shear rates, this motion is on the order of a few molecular diameters and so appears as no-slip at the macroscopic level. At higher shear rates, the motion becomes much more prevalent, on the order of tens or hundreds of nanometers, which can be observed macroscopically. Joshi et al. ${ }^{16,17}$ have developed a comprehensive model to predict slip in the flow of polymer melts over polymer surfaces.

The nature of the surface and the surface-liquid interaction also influences slip. Barrat and Bocquet ${ }^{18}$ used a molecular dynamics simulation to show slip behavior during the 
a)

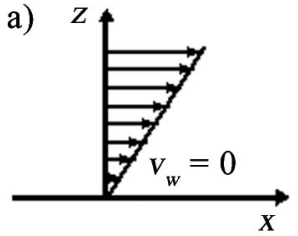

b)

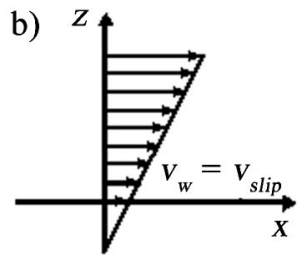

FIG. 1. Velocity profile for (a) the no-slip condition and (b) a slip condition with an imaginary no-slip location at some point in the wall.

wetting and dewetting of hydrophobic surfaces. Other molecular dynamics studies predict slip at high shear rates in a variety of configurations. ${ }^{19-22}$ Many experimental studies using atomic force microscopy or the surface force apparatus have noted slip of both hydrophobic and hydrophilic liquids on hydrophobic surfaces at high shear rates, with slip lengths on the order of tens or hundreds of nanometers. A recent study ${ }^{23}$ has even found slip of water at a hydrophilic interface at high shear rates $\left(10^{4} \mathrm{~s}^{-1}\right)$, with slip lengths on the order of $10 \mathrm{~nm}$. Thompson et al. ${ }^{24}$ suggested the possibility of slip at the interface of a TSM device operating in liquids, which was reiterated by Pit et al. ${ }^{25}$ This idea, however, has met with stiff resistance from the acoustic sensor community, which in many cases continues to use models with no-slip boundary conditions, such as the Sauerbrey model for attached mass and the Kanazawa and Gordon result for the response to liquid loading. ${ }^{2,26}$ While the no-slip boundary condition has been applied in many cases, it may not represent an accurate description for complex biomolecular adsorption in liquids. ${ }^{27}$

The motional resistance $R_{m}$ is another experimental value that can be measured along with the series resonant frequency, $f_{s}$. It is a measure of the amount of energy dissipated by any adsorbed mass or liquid at the surface, and can be thought of as due to the internal friction of a viscous or viscoelastic layer. $R_{m}$ is related to the dissipation factor, $D$, and its inverse $Q$, the filter quality factor.

Many experiments involving complex biomolecular interactions yield results that cannot be explained by the simple Sauerbrey mass model. ${ }^{2}$ Lyle et al. ${ }^{28}$ measured the binding of the drug warfarin to a chemisorbed layer of human serum albumin (HSA), attached to a TSM surface by different linker molecules. The binding of warfarin to HSA is known to alter the tertiary structure and possibly the hydrophobicity of the surface layer. This, in turn, could influence surface-liquid coupling and lead to possible slip behavior. In a recent experiment, ${ }^{29}$ light-activated cross-linking surface adsorbed monolayers (SAM) have been applied to the surfaces. Increases in the series resonant frequency $f_{s}$ and decreases in the motional resistance $R_{m}$ were observed, without any loss of the monolayer. Other similar situations have been reported in our laboratory. ${ }^{30,31}$

Slip can be applied to acoustic device models by introducing slip boundary conditions (BC), either as stress or displacement/velocity discontinuities. Rodahl and Kasemo ${ }^{32}$ used a shear stress BC where the difference in surface and liquid shear forces was modeled with a coefficient of friction $\chi$. This model was applied by McHale et al. ${ }^{33}$ in an $n$-layer impedance model, characterized by a slip parameter $s$. In this description, slip can be viewed as the feedback element in a single-loop negative feedback system, with the forward transfer function given by the no-slip impedance. Ferrante et al. ${ }^{34}$ and Hayward and Thompson ${ }^{35}$ used displacement slip BCs in two- and four-layer models of a quartz device, where slip was included as a multiplying factor $\alpha$ for the displacements of adjacent layers. The slip parameter $\alpha$ can be complex, so both magnitude and phase differences between layers can be modeled. Ellis and Hayward ${ }^{36}$ showed that, at a solid-liquid interface, the complex value $\alpha$ is related to the real-valued slip length by $\alpha=\cos (b / \delta \sqrt{j 2})+j \sin (b / \delta \sqrt{j 2})$. Furthermore, to a first-order approximation for small slip lengths, the slip parameter $s$ is related to the slip length $b$ by $s=b / \eta_{f}$ where $\eta_{f}$ is the liquid viscosity. ${ }^{36,37}$ This implies that, in certain circumstances, the stress and velocity boundary conditions are equivalent.

Despite the proliferation of experimental evidence and theoretical models for interfacial slip at solid-liquid boundaries, ${ }^{3-8,14-16,18,20,38-44}$ there is currently no method to predict the occurrence of slip, or its strength, on acoustic wave device surfaces. It is therefore difficult to determine whether interfacial slip is occurring or whether the acoustic responses are being influenced by other physical mechanisms. In an attempt to introduce the framework for such a model, we apply the Blake-Tolstoi treatment of molecular $\operatorname{slip}^{45,46}$ to the fundamental description of acoustic wave devices coupled to a liquid of finite thickness. This theory provides a description of slip that links a hydrodynamic slip boundary condition, to a schematic, molecular description involving the wettability of the liquid-solid interface.

\section{THEORY}

The following application of the slip boundary condition is based on an acoustic model that includes the hydrodynamic slip boundary condition and the Blake-Tolstoi description of molecular slip. ${ }^{45,46}$ It is summarized here for convenience, as well as to highlight some additions to the model.

\section{A. Slip on an acoustic device}

We begin with a finite liquid in contact with a solid surface oscillating in the shear direction at a frequency $\omega$ $=2 \pi f$, where $f$ is the resonant frequency of the combined system. The surface has a velocity of $\dot{q}_{s}$, which is determined by the properties of the solid and the liquid is of finite thickness $t_{f}$. This situation is shown in Fig. 2 .

The liquid velocity for a single fluid layer with no slip is, from McHale et al., ${ }^{33,37}$

$$
v_{f}(z)=\dot{q}_{s} \frac{\cosh \left[k_{f}\left(z-t_{f}\right)\right]}{\cosh \left[k_{f} t_{f}\right]},
$$

where $k_{f}$ is the liquid wave number $k_{f}=\sqrt{-2 j} / \delta, \delta$ is the shear wave decay length in the liquid $\delta=\sqrt{2 \eta_{f} / \omega \rho_{f}}$, and $\eta_{f}$ and $\rho_{f}$ are, respectively, the viscosity and density of the liquid and $\dot{q}_{s}$ is the speed of displacement of the substrate at the solid-liquid boundary. This gives the expected behavior of a damped sinusoidal shear wave in the liquid. 


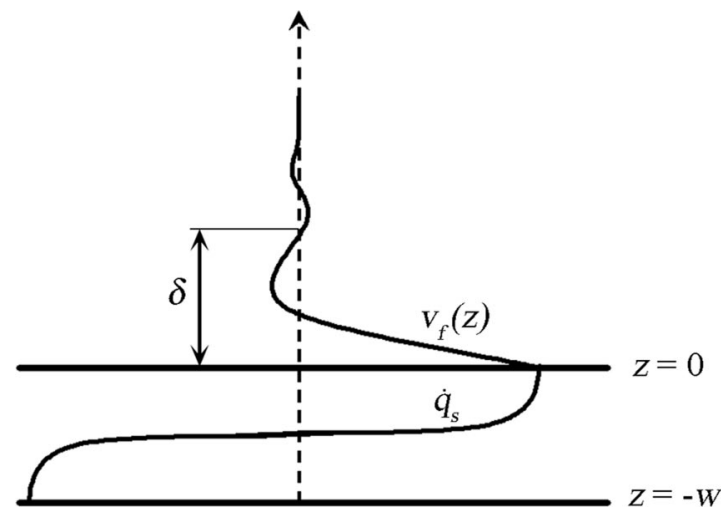

FIG. 2. No-slip velocity profile for a liquid layer of thickness $t_{f}$ on an oscillating surface with speed $\dot{q}_{s}$ and thickness $-w$. The decay length $\delta$ is the characteristic decay length of the damped oscillatory motion.

Normally, acoustic wave experiments involve the measurement of electrical properties, which can be linked to the acoustic impedance of the device. The impedance of the single-fluid layer device is

$$
Z_{f}^{\text {no-slip }}=\eta_{f} k_{f} \tanh \left(k_{f} t_{f}\right) .
$$

The change in series resonant frequency $f_{s}$ and damping $D$ can be estimated using the impedance from

$$
\begin{aligned}
& \Delta f_{s}=\frac{-1}{2 \pi \rho_{q} t_{q}} \operatorname{Im}\left(Z_{f}\right) \\
& \Delta D=\frac{2}{\omega \rho_{q} t_{q}} \operatorname{Re}\left(Z_{f}\right),
\end{aligned}
$$

where $\rho_{q}$ and $t_{q}$ are the density and thickness of the device substrate.

The slip length is included as

$$
v_{f}^{\operatorname{sip}}(-b)=\dot{q}_{s} .
$$

The velocity expression then becomes

$$
v_{f}^{\operatorname{slip}}(z)=\dot{q}_{s} \frac{\cosh \left[k_{f}\left(z-t_{f}\right)\right]}{\cosh \left[k_{f}\left(b+t_{f}\right)\right]} .
$$

From a first-order Taylor expansion, assuming that $b / \delta \ll 1$ and using Eq. (5), the velocity becomes

$$
v_{f}^{\operatorname{slip}}(z)=\dot{q}_{s} \frac{\cosh \left[k_{f}\left(z-t_{f}\right)\right]}{\cosh \left(k_{f} t_{f}\right)\left[1+Z_{f}^{\text {no-slip }} b / \eta_{f}\right]}
$$

and the liquid velocity at the wall is given by

$$
v_{f}^{\text {slip }}(z=0)=\frac{\dot{q}_{s}}{1+Z_{f}^{\text {no-slip }} b / \eta_{f}} .
$$

The acoustic impedance for the slip case becomes

$$
Z_{f}^{\text {slip }}=\frac{Z_{f}^{\text {no-slip }}}{1+Z_{f}^{\text {no-slip }} b / \eta_{f}} .
$$

From Eqs. (3) and (8), the acoustic properties can be calculated. The impedance can also be described using the slip parameter $s$, using the substitution $s=b / \eta_{f} \cdot{ }^{36,37}$
In the model explained below, Blake ${ }^{45}$ introduced a surface pressure term to account for the possibility that the boundary mobility may be less than the bulk. Inclusion of the surface pressure can result in negative slip lengths. McHale and Newton ${ }^{37}$ have shown that for small, negative slip lengths, Eq. (8) can provide an apparent "trapped mass" contribution to the frequency response of a TSM. Thus, a hydrodynamic slip boundary condition can be a suitable formulation to describe a rough, but completely wetted, TSM surface. However, a recent study ${ }^{47}$ has shown that positive boundary slip in fact increases with increasing surface roughness, as opposed to the negative slip length discuss above.

The "trapped gas" model is, however, a mathematical formulation, and there is no experimental evidence for trapped mass when using TSM devices with optically flat surfaces, as is the case for many devices. ${ }^{27}$ Molecular slip is a plausible alternative scenario. In the following section, we consider the situation of molecular mobility as it is related to slip at a smooth, partially wetting, TSM surface.

\section{B. Molecular mobility and surface tension}

The Tolstoi-Blake ${ }^{45,46}$ treatment of molecular slip is based on a molecular-kinetic description of mobility and diffusion of liquid molecules near a surface. We begin with Frenkel's ${ }^{48}$ description of the mobility of a liquid molecule of molecular diameter $\sigma$ and whose center is at a mean distance $r$ from the center of an adjacent molecule (mean intermolecular separation). Frenkel gave the mobility of a liquid molecule as:

$$
u=\frac{r^{2}}{6 k T \tau^{0}} e^{-W / k T},
$$

where $k$ is the Boltzmann constant and $T$ is the temperature, $\tau^{0}$ is the relaxation time of molecular displacement, and $W$ is the activation energy required to form a microcavity into which a neighboring molecule can move. The activation energy is $W=A \gamma^{\mathrm{LV}}$, where $\gamma^{\mathrm{LV}}$ is the surface tension of the liquid in a vacuum and $A$ is the effective molecular surface area. We use $A=\pi \sigma^{2}$ for a spherical molecule, distinguishing between the molecular diameter $\sigma$ and the center-tocenter molecular separation $r$. Tolstoi ${ }^{46}$ extended this idea to the layer of liquid molecules in contact with a solid boundary so that:

$$
u_{s}=\frac{r^{2}}{6 k T \tau_{s}^{0}} e^{-W_{s} / k T},
$$

where the subscript $s$ applies to the liquid molecules at the boundary. The activation energy for a liquid molecule at the boundary is given by the loss of the solid-liquid interfacial area and the gain in solid-vapor and liquid-vapor interfacial areas in opening a microcavity. Using $\alpha$ as the fraction of the microcavity area within the solid, we write

$$
\begin{aligned}
W_{s} & =\alpha A\left(\gamma^{\mathrm{SV}}-\gamma^{\mathrm{SL}}\right)+(1-\alpha) A \gamma^{\mathrm{LV}} \\
& =\alpha A\left(\gamma^{\mathrm{SV}}-\gamma^{\mathrm{SL}}-\gamma^{\mathrm{LV}}\right)+A \gamma^{\mathrm{LV}},
\end{aligned}
$$

where $\gamma^{\mathrm{SV}}$ and $\gamma^{\mathrm{SL}}$ are the surface tensions of the solid/ vacuum and solid/liquid interfaces. The factor $\alpha A$ must be estimated, but is expected to be on the order of $\sigma^{2}$. 


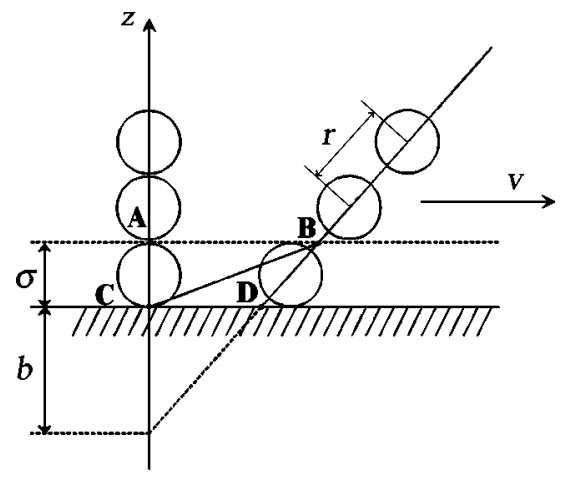

FIG. 3. Schematic representation of molecular slip from Blake (see Ref. 45) showing the motion of surface-adjacent liquid molecules in a velocity field. The particle diameter is $\sigma$ and the slip length is $b$.

A further approximation made by Tolstoi is that $\tau^{0}$ $=\tau_{s}^{0}$, so the ratio of surface to bulk mobility is

$$
\begin{aligned}
\frac{u_{s}}{u} & =\exp \left[\left(W-W_{s}\right) / k T\right] \\
& =\exp \left[\alpha A\left(\gamma^{\mathrm{SL}}+\gamma^{\mathrm{LV}}-\gamma^{\mathrm{SV}}\right) / k T\right] .
\end{aligned}
$$

The difference in activation energies depends on the interfacial energies through $\alpha A\left(\gamma^{\mathrm{SL}}+\gamma^{\mathrm{LV}}-\gamma^{\mathrm{SV}}\right)$. By using Young's Law $\gamma^{\mathrm{SV}}-\gamma^{\mathrm{SL}}=\gamma^{\mathrm{LV}} \cos \theta$, where $\theta$ is the equilibrium contact angle of a liquid droplet on a solid surface in vapor, Eq. (12) can be written

$$
\frac{u_{s}}{u}=\exp \left[\alpha A \gamma^{\mathrm{LV}}(1-\cos \theta) / k T\right] .
$$

This theory predicts that the mobility of liquid molecules at the boundary between a liquid and a solid is the same as in the bulk liquid when the surface is completely wetted $\left(\theta \rightarrow 0^{\circ}\right)$, but that a partially wetting surface $\left(\theta>0^{\circ}\right)$ results in liquid molecules at the boundary being more mobile than in the bulk phase. Since the mobility of a molecule is the average velocity with respect to surroundings when acted upon by a force of unit magnitude, we anticipate that the same shear stress will result in different velocity gradients across at least the first molecular layer in contact with the solid compared to those in the liquid.

\section{Molecular slip}

From continuum fluid mechanics the slip velocity $\Delta v_{s}$ induced by a shear stress $\tau$ is $\Delta v_{s}=\tau / \kappa$, where $\tau$ is the shear stress and $\kappa$ is the liquid-solid coefficient of friction ${ }^{3,32,33}$ and $\Delta v_{s}$ is the discontinuous change in velocity at the mathematical surface representing the wall. It is evident that for infinite friction, there is no slip, and for no friction, there is infinite slip. The molecular situation is shown in Fig. 3. From the shear stress in a viscous liquid:

$$
\tau=\eta_{f}\left(\frac{d v}{d z}\right)=\kappa \Delta v_{s},
$$

and the trigonometry of Fig. 3 which gives $\Delta v_{s}=\mathrm{CD}$ $=b(d v / d z)$, we deduce,

$$
b=\frac{\eta_{f}}{\kappa} .
$$

Equation (15) is the result commonly used by de Gennes and others. ${ }^{3,4,6,42}$ It is interesting to note that the friction coefficient $\kappa$ is equivalent to the slip parameter $s=\eta_{f} / b$ used by McHale et al. ${ }^{33}$

The result in Eq. (15) is a continuum result. To complete the development, a molecular description is required. The molecular mobility at the surface is related to the motion of particles near that surface. For a bulk shear rate of $(d v / d z)$ induced by the shear stress $\tau$, the velocity of a molecule relative to its neighbor would be $r(d v / d z)$, where $r$ is the mean distance to the center of an adjacent molecule. The force on the molecule would then be $r^{2} \tau$ and the mobility is

$$
u=\frac{1}{r \tau}\left(\frac{d v}{d z}\right) .
$$

For molecules near the surface, with the presence of slip, we would replace the bulk shear rate with a different boundary shear rate $(d v / d z)_{s}$, still for a given $\tau$. The velocity gradient near the surface was described schematically by Tolstoi, and is shown in Fig. 3. It shows that

$$
\frac{u_{s}}{u}=\frac{(d v / d z)_{s}}{(d v / d z)}=\frac{A B / r}{A B /(b+r)}=\frac{b}{r}+1 .
$$

Therefore, the slip length used in the hydrodynamic model for acoustic wave devices is related to the ratio of boundary to bulk mobility by $b=r\left(u_{s} / u-1\right)$. Figure 3 illustrates a molecular description of the hydrodynamic slip boundary condition shown in Fig. 1. Rearranging Eq. (17) and inserting Eq. (13), we find

$$
b=r\left\{\exp \left[\alpha A \gamma^{\mathrm{LV}}(1-\cos \theta) / k T\right]-1\right\},
$$

which is the result obtained of Tolstoi and Blake. Equation (18) is a key result because it links the hydrodynamic equations for a TSM device using a slip length boundary condition to the wettability of the solid surface characterized by the contact angle $\theta$. It is reassuring that slip lengths predicted from this model are on the order of molecular dimensions, since the exponent term is multiplied by the molecular diameter. Equation (18) predicts that the slip length vanishes for a completely wetting liquid $\left(\theta \rightarrow 0^{\circ}\right)$, but becomes exponentially important as increasingly nonwetting liquids are used, with the most extreme case when $\left(\theta \rightarrow 180^{\circ}\right)$. For the case of water, the theory predicts that a no-slip boundary condition is expected for a hydrophilic TSM surface, but that slip could occur on a highly hydrophobic TSM surface. It has been reported in the literature that slip may occur for water on hydrophilic surfaces. ${ }^{23}$ However, the current theory does not predict this.

Assuming a spherical liquid molecule, from Eq. (18), the slip length can be estimated from the literature and experimental data related to the contact angle. Moreover, based on knowledge of the contact properties between a liquid and a solid, we can approximate the behavior of an acoustic wave device in liquid, using Eqs. (3), (8), and (18). This is a direct predictive model for slip behavior on an acoustic wave device that links surface and contact properties to acoustic 


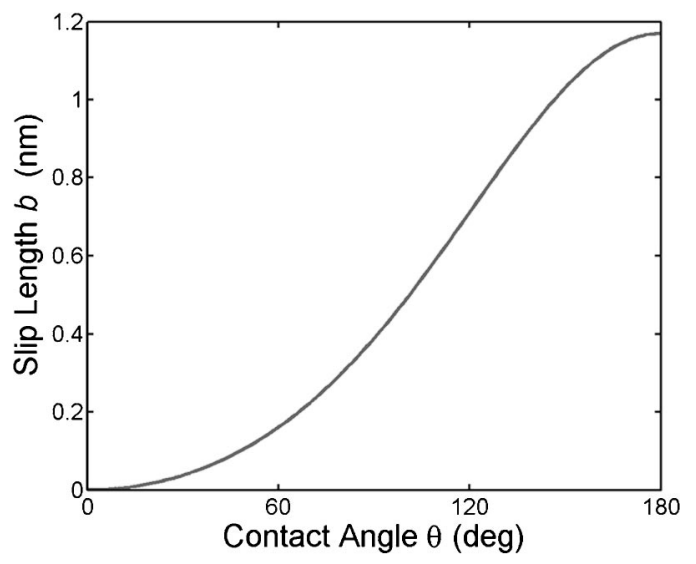

FIG. 4. Change in slip length $b$ as contact angle varies from $0^{\circ}$ to $180^{\circ}$, with microcavity fraction $\alpha=1 / 6$, molecular diameter $\sigma=0.276 \mathrm{~nm}$, the approximate size of water, and $r=0.385 \mathrm{~nm}$, the mean separation, as estimated from the density of water at $25{ }^{\circ} \mathrm{C}$, assuming a spherical molecule.

properties. In the following sections, we explore the behavior of the model over a range of contact and surface values, and then discuss its physical limitations and potential applications.

\section{MODEL BEHAVIOR}

To assess this slip length model, we will evaluate its behavior over a range of situations. The dependence of slip length on contact angle shows the expected exponential dependence, with low slip values for completely wetting films, followed by a rapid increase for partially wetting liquid, and then flattening out for nonwetting films. This is shown in Fig. 4 for water $\left[\gamma^{\mathrm{LV}}\left(25^{\circ} \mathrm{C}\right)=72.0 \mathrm{mN} / \mathrm{m}, \quad \sigma \approx 2.76 \AA, \quad r\right.$ $\approx 3.85 \AA$ ] over a range of contact angles, which is experimentally equivalent to varying the interaction of the surface with the water. This value is the surface tension for water in a vacuum, ${ }^{49}$ which may deviate slightly from the surface tension of water in its vapor, but the difference would be small, and of little significance to our results.

While this situation demonstrates the expected qualitative behavior of the model, it predicts slip lengths lower by at least an order of magnitude than those deduced from ex-

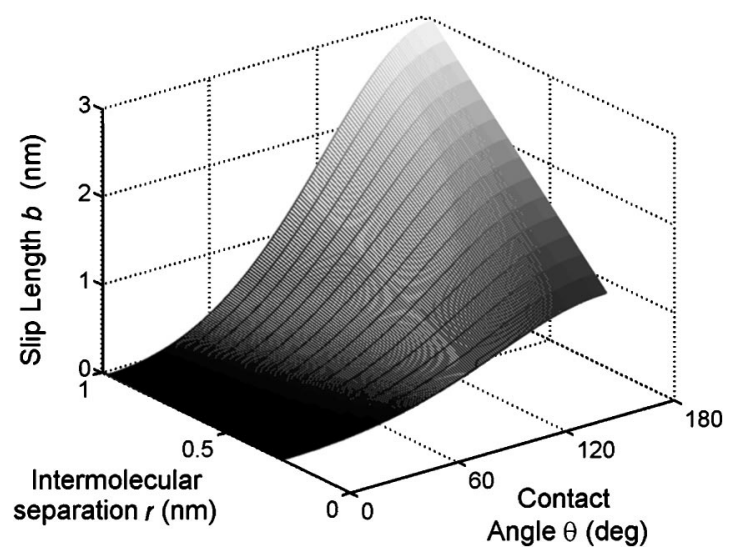

FIG. 5. Change in slip length $b$ as contact angle varies from $0^{\circ}$ to $180^{\circ}$ and intermolecular separation $r$ from 2.76 to $9 \AA$, with molecular diameter $\sigma=2.76 \AA$ and microcavity fraction $\alpha=1 / 6$.

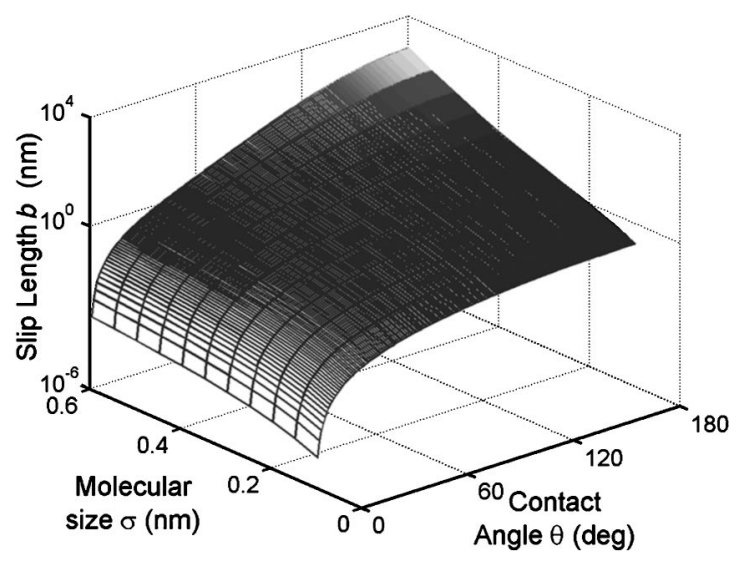

FIG. 6. Change in slip length $b$ as contact angle varies from $0^{\circ}$ to $180^{\circ}$ and molecular diameter $\sigma$ varies from 1 to $6 \AA$, with microcavity fraction $\alpha=1 / 6$ and mean intermolecular separation $r=9 \AA$. The scale is shown logarithmically to better illustrate the changes in slip length.

perimental observations, both on a $\mathrm{TSM}^{36}$ and in other systems. ${ }^{14,41,43,50}$ This is due to the sensitivity of the model to the molecular diameter $\sigma$, the mean separation $r$, and the fraction of the microcavity within the solid $\alpha$.

The liquid would not behave as close-packed particles, so using the same value for both the molecular size $\sigma$ and intermolecular separation $r$ would not be realistic. Figure 5 shows the change in slip length for various values of $r$, including that of water. Since the mean separation enters the model in Eq. (18) as a proportionality factor, it does not greatly influence the behavior, and values for slip length are still low in this model. However, caution must be exercised in treating $r$ solely as a proportionality factor, since $r$ likely enters the exponent in the factor $\alpha A$. This point is discussed further in the following section.

Figure 6 shows the change in slip length with molecular diameter $\sigma$, which appears in the model within the exponent. It therefore has a strong effect on the slip length, and produces physically realistic values when $\sigma \approx 5 \AA$. Figure 7 shows the variation in slip length with $\alpha$, which is the fraction of the molecular cavity that is solid. The shape of Fig. 7 is similar to that of Fig. 6, since both $\sigma$ and $\alpha$ enter the model in the exponent. However, the slopes are different,

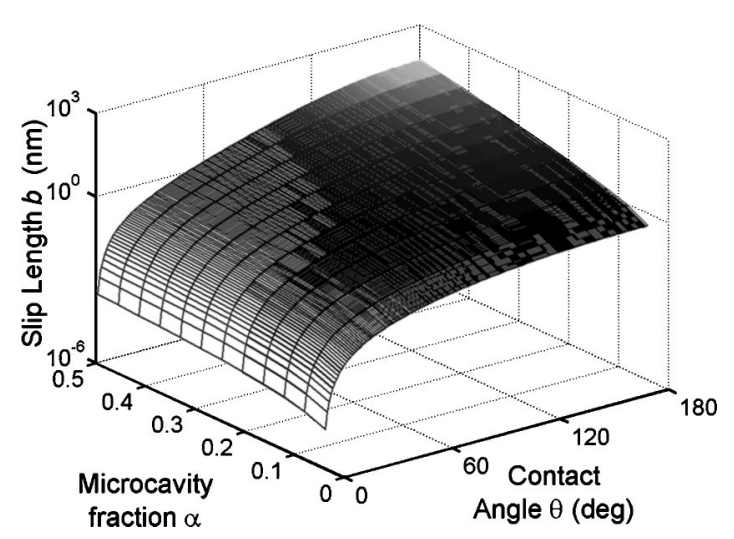

FIG. 7. Change in slip length $b$ as contact angle varies from $0^{\circ}$ to $180^{\circ}$ and microcavity fraction $\alpha$ varies from 0 to $1 / 2$, with molecular diameter $\sigma=2.76 \AA$ and intermolecular separation $r=9 \AA$. 


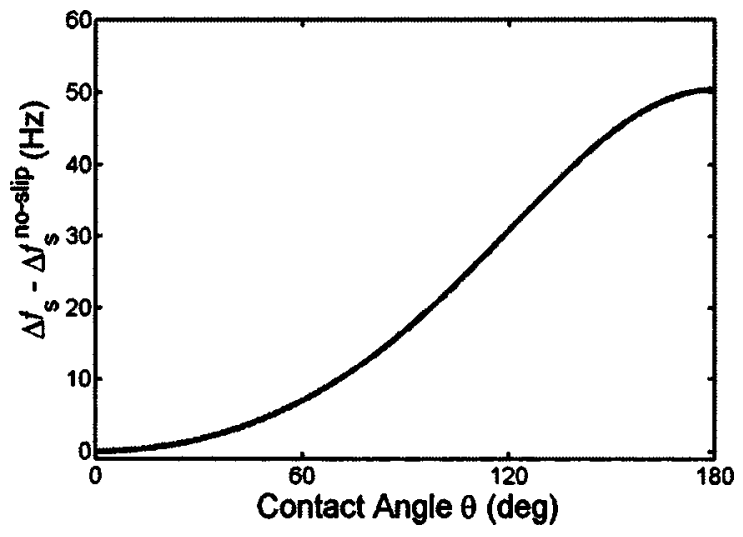

FIG. 8. Change in frequency shift with contact angle, compared to the no-slip condition. The contact angle varies from $0^{\circ}$ to $180^{\circ}$, for microcavity fraction $\alpha=1 / 6$, molecular diameter $\sigma=6 \AA$, and intermolecular separation $r=9 \AA$.

since $\sigma$ is squared in the area, and thus has a greater effect. Blake did not offer a method for determining the microcavity fraction $\alpha$, though it may be analogous to calculating the contact area for an elastic sphere in contact with a solid surface.

In the analysis of Figs. 5, 6, and 7, $r$ and $\sigma$ were assumed independent. It is important to note that this is likely not the case, since the separation between polar molecules depends on the interaction between them, as well as the size of the individual molecules. While an analysis of this relationship is essential for the complete characterization of the predictive model, it is very rigorous, and will not be covered here. Since the intermolecular separation $r$ only enters the model as a linear factor, for the remainder of this study, we treat $r$ and $\sigma$ independently. For a more complete coverage of intermolecular forces and separations, see for example Ref. 51.

Figures 8 and 9 show the changes in resonant frequency and motional resistance with contact angle, as calculated from Eqs. (3) and (8), compared to the shifts with the no-slip condition. Frequency shifts are displayed in $\mathrm{Hz}$, so Fig. 8 shows a frequency increase with increases in contact angle. The model shows the expected dependence of both series resonant frequency and motional resistance on contact angle.

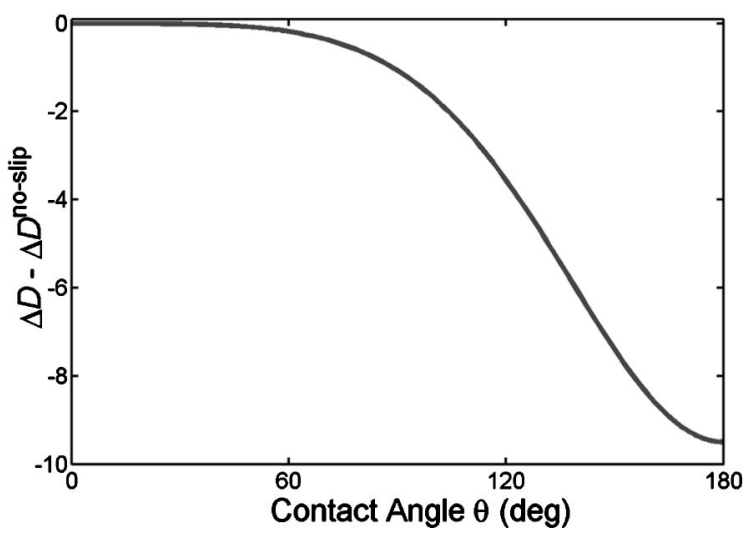

FIG. 9. Change in dissipation with contact angle, compared to the no-slip condition. The contact angle varies from $0^{\circ}$ to $180^{\circ}$, for microcavity fraction $\alpha=1 / 6$, molecular diameter $\sigma=6 \AA$, and intermolecular separation $r=9 \AA$.

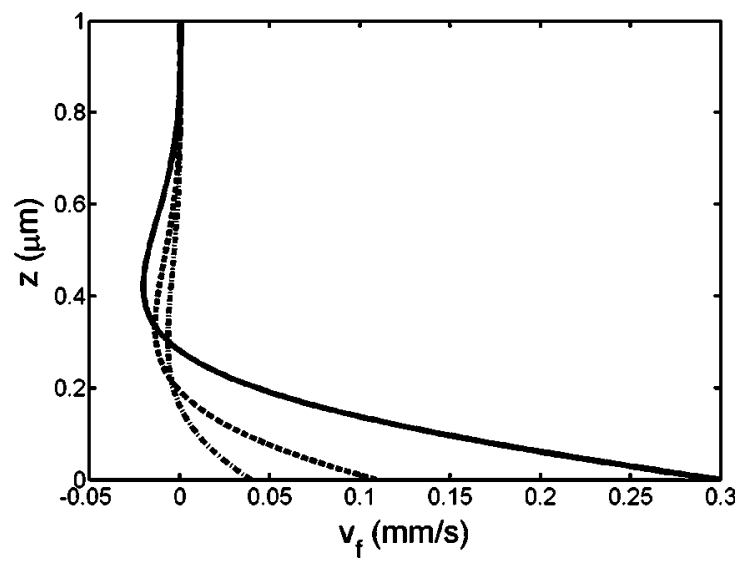

FIG. 10. Liquid velocity profile for three different contact angles, with a surface velocity of $300 \mu \mathrm{m} / \mathrm{s}$ : (a) solid line $\left(0^{\circ}\right)$ shows the no-slip case, (b) dashed $\left(130^{\circ}\right)$ shows some slip, and (c) dash-dot $\left(180^{\circ}\right)$ shows greater slip. Here, the microcavity fraction $\alpha=1 / 6$, molecular diameter $\sigma=6 \AA$, and intermolecular separation $r=9 \AA$.

The slip length of $b=0$ corresponds to the no-slip condition, which are the shifts in frequency and dissipation for a surface covered with a $100-\mu \mathrm{m}$-thick film of water, with density $997 \mathrm{~kg} / \mathrm{m}^{3}$ and viscosity $0.914 \mathrm{cP}$, at a surface speed of 0.3 $\mathrm{mm} / \mathrm{s}$. As the contact angle increases, indicating a decrease in interfacial coupling, the resonant frequency shift and the dissipation both decrease. This is because less energy is lost through viscous interactions with the liquid as the coupling decreases. The resonant frequency responds faster to changes in coupling, which is observed in a similar slip model, ${ }^{33}$ indicating that frequency is a more sensitive indicator of surface coupling than dissipation.

The shear rate in the liquid is found from the velocity profile, which decays rapidly with decay length $\delta$. When slip is introduced, the velocity at the wall is reduced, as shown in Fig. 10 for a $100-\mu$ m-thick film of water, with density $997 \mathrm{~kg} / \mathrm{m}^{3}$ and viscosity $0.914 \mathrm{cP}$, at a surface speed of 0.3 $\mathrm{mm} / \mathrm{s}$. Here, $r=9 \AA, \sigma=6 \AA$ and $\alpha=1 / 6$.

These situations deal solely with the surface-liquid interface, where the surface layer is treated as a pure solid and the liquid as mobile. In biosensor applications, there are always biomolecule and linker layers present that act as the signal transducers as surface-layer reactions proceed. Binding events may alter the wettability, which in turn would affect the slip length, either increasing or decreasing it. However, there are other factors that can also play a role in slip. The internal structure, most importantly the viscoelasticity of the linker and biomolecule layers, will affect the relative velocity at the solid-liquid interface. ${ }^{4}$ This does not refer to the bulk acoustic properties of the film, but the stiffnessinduced hydrodynamic properties of the interface, which is shown to be a factor in oscillatory flow in nonequilibrium molecular dynamics studies. ${ }^{20}$

\section{CONCLUSIONS}

We have revisited the Blake-Tolstoi theory for molecular and hydrodynamic slip and applied it to the surfaceliquid interface of an acoustic wave sensor in liquid. For a complex biomolecular surface, understanding the relation- 
ship between hydrophobicity and acoustic wave response may give insight into conformation and structure changes at the surface. The current theory shows the expected qualitative behavior of a predictive model for slip. Although not a complete quantitatively predictive model, physically reasonable results are obtained by adjusting the particle size and surface microcavity fraction. It is important to note, however, that the manipulation of particle size and surface microcavity size is not physically realistic and it must be emphasized that this was only done to demonstrate the qualitative behavior of the model.

There are significant limitations to the above theory, many of which are discussed by Tolstoi and Blake. The foremost of these is the difficulty in estimating the microcavity fraction $\alpha$. This was noted by Tolstoi, who simply assumed $\alpha=1 / 6$ and $1 / 9$ to correspond to experimental data. In doing so, $\alpha$ becomes another fitting parameter. Another limitation is that Frenkel's original approach is schematic, and therefore phenomenological. A more mathematically rigorous diffusion treatment may yield more realistic results. In addition, assuming $\tau^{0}=\tau_{b}^{0}$ is a rough approximation. Due to molecular ordering of the surface, the relaxation times of surface versus bulk velocities may be significantly different. With a more rigorous treatment of the diffusion, and a better understanding of the particle size and microcavity function, the TolstoiBlake model may offer a quantitative method for predicting dynamic interactions at the surface-liquid interface of acoustic wave devices.

\section{ACKNOWLEDGMENTS}

J.S.E. would like to thank Dr. David Stone for his helpful discussion. J.S.E., M.T., and G.L.H. also gratefully acknowledge the Natural Sciences and Engineering Research Council and Ontario Graduate Scholarships for financial support. G.M. wishes to acknowledge the UK EPSRC for financial support under Grant No. GR/S30573/01 (2003).

${ }^{1}$ B. A. Cavic, G. L. Hayward, and M. Thompson, Analyst (Cambridge, U.K.) 124, 1405 (1999).

${ }^{2}$ G. Sauerbrey, Z. Phys. 155, 206 (1959).

${ }^{3}$ L. Leger, H. Hervet, G. Massey, and E. Durliat, J. Phys.: Condens. Matter 9, 7719 (1997)

${ }^{4}$ L. Leger, H. Hervet, and G. Massey, Trends Polym. Sci. 5, 40 (1997).

${ }^{5}$ F. Brochard and P. G. de Gennes, Langmuir 8, 3033 (1992).

${ }^{6}$ F. Brochard-Wyart, H. Hervet, and C. Redon, Abstr. Pap. - Am. Chem. Soc. 209, 13 (1995).

${ }^{7}$ P.-G. de Gennes, Soft Interfaces: The 1994 Dirac Memorial Lectures (Cambridge University Press, Cambridge, 1997).

${ }^{8}$ K. B. Migler, H. Hervet, and L. Leger, Phys. Rev. Lett. 70, 287 (1993).

${ }^{9}$ J. Israelachvili, J. Vac. Sci. Technol. A 10, 2961 (1992).

${ }^{10}$ J. N. Israelachvili, J. Colloid Interface Sci. 110, 263 (1986).

${ }^{11}$ T. D. Blake, A. Clarke, J. DeConinck, and M. J. deRuijter, Langmuir 13, 2164 (1997).
${ }^{12}$ T. D. Blake and J. De Coninck, Adv. Colloid Interface Sci. 96, 21 (2002).

${ }^{13}$ T. D. Blake, C. Decamps, J. De Coninck, M. de Ruijter, and M. Voue, Colloids Surf., A 154, 5 (1999).

${ }^{14}$ V. S. J. Craig, C. Neto, and D. R. M. Williams, Phys. Rev. Lett. 8705, 054504 (2001).

${ }^{15}$ Y. X. Zhu and S. Granick, Phys. Rev. Lett. 8709, 096105 (2001).

${ }^{16}$ Y. M. Joshi, A. K. Lele, and R. A. Mashelkar, J. Non-Newtonian Fluid Mech. 94, 135 (2000).

${ }^{17}$ Y. M. Joshi, A. K. Lele, and R. A. Mashelkar, Macromolecules 34, 3412 (2001).

${ }^{18}$ J. L. Barrat and L. Bocquet, Phys. Rev. Lett. 82, 4671 (1999).

${ }^{19}$ S. A. Gupta, H. D. Cochran, and P. T. Cummings, J. Chem. Phys. 107, 10316 (1997)

${ }^{20}$ A. Jabbarzadeh, J. D. Atkinson, and R. I. Tanner, J. Chem. Phys. 110, 2612 (1999)

${ }^{21}$ A. Jabbarzadeh, J. D. Atkinson, and R. I. Tanner, Phys. Rev. E 61, 690 (2000).

${ }^{22}$ A. Jabbarzadeh, J. D. Atkinson, and R. I. Tanner, Tribol. Int. 35, 35 (2002).

${ }^{23}$ E. Bonaccurso, M. Kappl, and H. J. Butt, Phys. Rev. Lett. 88, 076103 (2002).

${ }^{24}$ M. Thompson, G. K. Dhaliwal, C. L. Arthur, and G. S. Calabrese, IEEE Trans. Ultrason. Ferroelectr. Freq. 34, 127 (1987).

${ }^{25}$ R. Pit, H. Hervet, and L. Leger, Tribol. Lett. 7, 147 (1999).

${ }^{26}$ K. K. Kanazawa and J. G. Gordon, Anal. Chem. 57, 1770 (1985).

${ }^{27}$ M. Thompson, R. Nisman, G. L. Hayward, H. Sindi, A. C. Stevenson, and C. R. Lowe, Analyst (Cambridge, U.K.) 125, 1525 (2000).

${ }^{28}$ E. L. Lyle, G. L. Hayward, and M. Thompson, Analyst (Cambridge, U.K.) 127, 1596 (2002).

${ }^{29}$ E. L. Lyle (Private communication).

${ }^{30}$ C. N. Jayarajah and M. Thompson, Biosens. Bioelectron. 17, 159 (2002).

${ }^{31}$ N. Tassew and M. Thompson, Anal. Chem. 74, 5313 (2002).

${ }^{32}$ M. Rodahl and B. Kasemo, Sens. Actuators A 54, 448 (1996).

${ }^{33}$ G. McHale, R. Lucklum, M. I. Newton, and J. A. Cowen, J. Appl. Phys. 88, 7304 (2000)

${ }^{34}$ F. Ferrante, A. L. Kipling, and M. Thompson, J. Appl. Phys. 76, 3448 (1994).

${ }^{35}$ G. L. Hayward and M. Thompson, J. Appl. Phys. 83, 2194 (1998).

${ }^{36}$ J. S. Ellis and G. L. Hayward, J. Appl. Phys. (submitted).

${ }^{37}$ G. McHale and M. I. Newton (unpublished).

${ }^{38}$ F. Baldoni, Z. Angew. Math. Mech. 79, 193 (1999).

${ }^{39}$ H. A. Barnes, J. Non-Newtonian Fluid Mech. 56, 221 (1995).

${ }^{40}$ E. Durliat, H. Hervet, and L. Leger, Europhys. Lett. 38, 383 (1997).

${ }^{41}$ R. G. Horn, O. I. Vinogradova, M. E. Mackay, and N. Phan-Thien, J. Chem. Phys. 112, 6424 (2000).

${ }^{42}$ R. Pit, H. Hervet, and L. Leger, Abstr. Pap. - Am. Chem. Soc. 218, 232 (1999).

${ }^{43}$ R. Pit, H. Hervet, and L. Leger, Phys. Rev. Lett. 85, 980 (2000).

${ }^{44}$ O. I. Vinogradova, Int. J. Min. Process 56, 31 (1999).

${ }^{45}$ T. D. Blake, Colloids Surf. 47, 135 (1990).

${ }^{46}$ D. M. Tolstoi, Dokl. Akad. Nauk SSSR 85, 1329 (1952)

${ }^{47}$ E. Bonaccurso, H.-J. Butt, and V. S. J. Craig, Phys. Rev. Lett. 90, 144501 (2003).

${ }^{48}$ I. A. I. I. Frenkel, Kinetic Theory of Liquids (Clarendon, Oxford, 1946).

${ }^{49}$ D. R. e. Lide, CRC Handbook of Chemistry and Physics, 73rd ed. (CRC, Boca Raton, FL, 1993).

${ }^{50}$ C. Cottin-Bizonne, S. Jurine, J. Baudry, J. Crassous, F. Restagno, and E. Charlaix, Eur. Phys. J. E 9, 47 (2002).

${ }^{51}$ J. N. Israelachvili, Intermolecular and Surface Forces, 2nd ed. (Academic, London, 1991). 